\title{
Searching for Time Reversal Invariance Violation in Polarized Neutron Decay
}

\author{
L.J. Lising*, J.M. Adams ${ }^{\star}$, J.M. Anaya ${ }^{\ddagger}$, T.J. Bowles ${ }^{\ddagger}$, T.E. \\ Chupp $^{* *}$, K.P. Coulter ${ }^{* *}$, M.S. Dewey ${ }^{\star}$, S.R. Elliott ${ }^{\dagger}$, S.J. \\ Freedman*, B.K. Fujikawa ${ }^{*}$, A. Garcia ${ }^{a}$, G.L. Greene ${ }^{\ddagger}$, S.-R. \\ Hwang $^{* *}$, G.L. Jones ${ }^{\star}$, J.S. Nico ${ }^{\star}$, H.G.R. Robertson ${ }^{\dagger}$, T.D. \\ Steiger $^{\dagger}$, W.A. Teasdale ${ }^{\ddagger}$, A.K. Thompson ${ }^{\star}$, E.G. Wasserman ${ }^{* b}$, \\ F.E. Wietfeldt ${ }^{\star}$, and J.F. Wilkerson ${ }^{\dagger}$
}

*Lawrence Berkeley National Laboratory Berkeley, CA 94720

$\dagger$ University of Washington, Seattle, WA, 98195

* National Institute of Standards and Technology, Gaithersburg, MD 20899

** University of Michigan, Ann Arbor, MI 48109

$\ddagger$ Los Alamos National Laboratory, Los Alamos, NM, 87545

${ }^{a}$ University of Notre Dame, Notre Dame, IN 46556

${ }^{b}$ Present address: Abacus Concepts, Inc., Berkeley, CA, 94704

\begin{abstract}
Time reversal invariance violation is tightly constrained in the Standard Model, and the existence of a T-violating effect above the predicted level would be an indication of new physics. A sensitive probe of this symmetry in the weak interaction is the measurement of the $D$-coefficient in neutron decay. This parameter characterizes the triple-correlation of neutron spin, electron momentum, and neutrino (or proton) momentum, which changes sign under time reversal. The emiT experiment, now on line, attempts to improve the measurement of $D$, whose current average is $0.3 \pm 1.5 \times 10^{-3}$.
\end{abstract}

\section{INTRODUCTION}

The origin of CP-violation has been a mystery since its observation in the kaon system in 1964. [1] The elusive nature of this phenomenon is compounded by the lack of any other evidence of CP-violation, or any direct observation of T-violation, which is implied under conservation of CPT. [2] An explanation within the Standard Model, via a phase in the Cabbibo-Kobayashi-Maskawa matrix of quark mixing, arises as a natural consequence of three generations 
of quarks. [3] While this formulation is adequate to describe the observations to date in kaons, it cannot address other suggestions of T-noninvariance such as Sakharov's mechanism for the evolution of the observed matter-antimatter asymmetry in the universe today. [4]

The neutron, a natural laboratory for precision studies for its neutrality and simplicity, presents several T-violating observables for which the Standard Model predicts values so small as to be experimentally inaccessible, yet could be measurable in alternative theories. Among the quantities are terms in the decay probability such as the "triple correlation",

$$
D \hat{\sigma}_{n} \cdot \mathrm{p}_{\mathrm{e}} \times \mathrm{p}_{\mathbf{p}}
$$

Previous attempts to measure the $\mathrm{D}$-coefficient have been consistent with zero with an average value of $0.3 \pm 1.5 \times 10^{-3}$. [5,6] The interpretation of correlations is usually complicated by the presence of final state effects, whereby $\mathrm{T}$-invariant interactions mimic the $\mathrm{T}$-violating signal at higher orders. However, for the neutron, the value of $D$ from this effect is calculated to be near $5 \times 10^{-5},[7,8]$ leaving room for further exploration.

Several proposed extensions to the Standard Model allow a D-coefficient above the final state effects. [9] These include models with left-right symmetry which incorporate a heavy, right-handed W-boson, which can lead to T-violation if the boson mass eigenstates mix the two chiralities. Models with new, "exotic" fermions can similarly mix with their lighter counterparts. Models that allow quark to lepton transitions via "leptoquark" bosons can also show interference between the leptoquarks that couple to the electrons and neutrinos. Both the exotic fermions and L-R symmetry can lead to other T-violating effects, such as the kaon $\epsilon^{\prime} / \epsilon$ and the neutron electric dipole moment, both of which have been measured with great precision. Within the context of these models, one can estimate the expected contribution to the D-coefficient to be less than $3 \times 10^{-5}$, although this requires certain assumptions. However, the leptoquark prediction for $D$ is not limited by these other two measurements as the leptoquark contribution to non-leptonic processes is not of lowest order.

\section{EXPERIMENTAL METHOD}

The emiT experiment is presently running at the National Institute of Standards and Technology's Cold Neutron Research Facility in Gaithersburg, Md. The facility utilizes a liquid hydrogen cold source to moderate thermal reactor neutrons, delivering them to users with minimal loss and a low gamma background by transport via reflecting guide tubes lined with $\mathrm{Ni}-58$. A polarization of $96 \%$ is obtained by state-selective reflection inside a supermirror polarizer and is retained along a 5 gauss alignment field through the spin flipper and 
collimator regions into the detector. The detector consists of an octagonal array of four each proton and electron detectors as shown below.

The octagonal geometry maximizes the experiment's sensitivity to $\mathrm{D}$ by balancing the sine dependence of the cross product with the large angles favored by kinematics. The symmetry of the detector and flipping of the neutron spin every few seconds reduces our sensitivity to varying detector efficiencies and fluctuations in the beam, which cancel to first order in the extraction of $D$ from the data. The electron segments are $50 \mathrm{~cm}$ long, 1/4-inch thick plastic scintillators with bialkali phototubes at each end. The recoil protons, whose maximum energy is only $750 \mathrm{eV}$, drift in a field free region until they near one of the four proton detectors, where they are accelerated through 36 kilovolts onto an array of windowless PIN diodes. The characteristic delay time of $0.5 \mu s-2.0 \mu s$ between the recoil proton and electron pulses is used to distinguish signal from beam-related background. Careful study and modelling of systematics have allowed us to minimize and monitor the factors that could cause a false measurement of $D$. The two primary effects are due to deviations of the polarization from purely longitudinal, and to nonuniformities in detection efficiency over the face of the detectors. [10]

\section{FIRST RUN}

The first of our allotted reactor cycles was devoted to beamline development, including alignment and polarization measurements. The neutron flux was measured at the end of the neutron guide (before the polarizer) to be $1 \times 10^{9}$ neutrons/second, and $1 \times 10^{8} \mathrm{n} / \mathrm{s}$ at the end our collimator series into the detector chamber. Following that, some time was devoted to testing

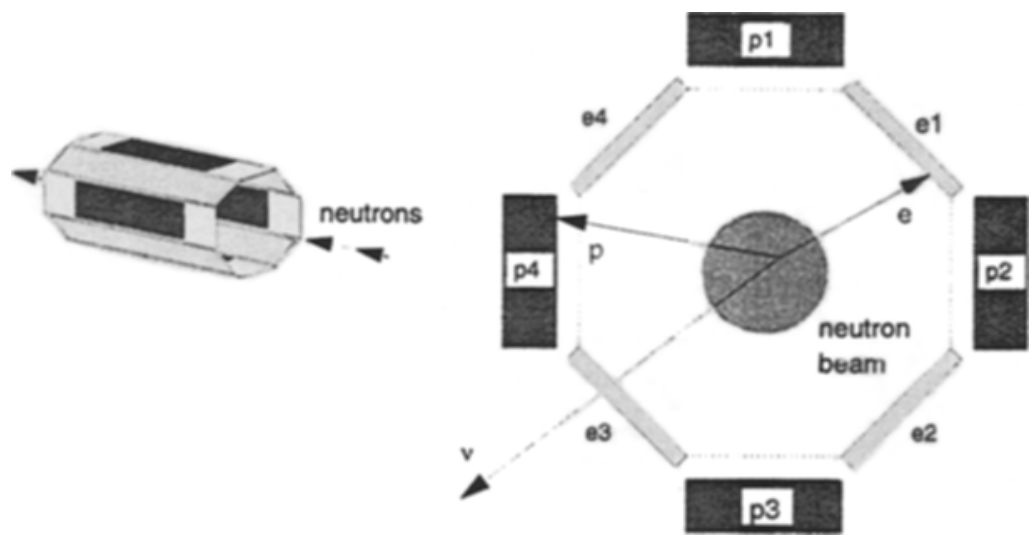

FIGURE 1. Detector geometry. Proton and electron detectors surround a $6 \mathrm{~cm}$ diameter longitudinally polarized beam. 
and adaptaion of our data acquisition system, noise and background reduction, vacuum improvement, and most importantly, stabilization and control of the high voltage, including protection of electronics from spark damage and minimization of field emission background. The ratio of signal to background in the correct timing window varies widely depending on noise conditions, yet the background is reduced to less than $1 / 10$ of the signal once the proper energy constraints are applied.

The expected rate for the full detector was $5-10 \mathrm{~Hz}$ in accepted neutron decays. We have already seen rates above $5 \mathrm{~Hz}$ and will recover more with repairs and improvements, which are ongoing. Analysis of the data is in progress, so far yielding a D-coefficient consistent with zero.

\section{REFERENCES}

1. Christenson, J. H. et al.,Phys. Rev. Lett. 13(4), 138-140 (1964).

2. Luders, G., Ann. Phys. 2, 1-15(1957).

3. Kobayashi, M., and Maskawa, T., Prog. Theo. Phys. 49(2), 652-657 (1973).

4. Sakharov, A., JETP Lett. 5, 24-27 (1967).

5. Steinberg, R.I. et al., Phys. Rev. Lett. 33(1), $41-44$ (1974).

6. Erozolimskii, B.G. et al., Sov. J. Nucl. Phys. 28, 48 (1978).

7. Jackson, J.D., Treiman, S.B., and Wyld, H.W.,Nucl. Phys. 4, 206-212 (1957).

8. Callan, C.G. and Treiman, S.B., Phys. Rev. 162(5), 1494-1497 (1962).

9. Herczeg, P., in Progress in Nuclear Physics, Hwang, W.-Y.P., Ed., Elsevier Science Publishing Co., Inc., 1991, pp. 171-194.

10. Wasserman, E.G., Ph.D. Thesis (1994).

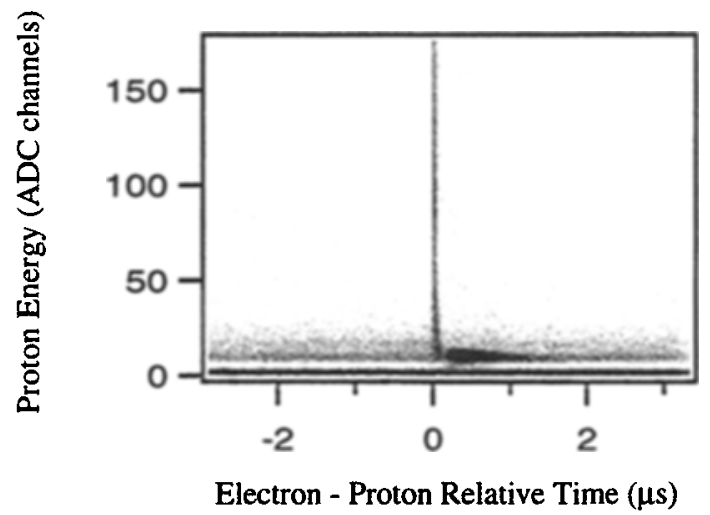

FIGURE 2. Proton detector energy versus relative electron-proton signal arrival time. The drift-delayed proton signal is well-separated from the narrow, high energy band of coincident events at zero time. 